\title{
A simple approach to evaluate the ergodic capacity and outage probability of correlated Rayleigh diversity channels with unequal signal-to-noise ratios
}

\author{
Muhammad Moinuddin ${ }^{1}$ and Imran Naseem²*
}

\begin{abstract}
In this article, we propose a novel method to derive exact closed-form ergodic capacity and outage probability expressions for correlated Rayleigh fading channels with receive diversity. Unlike the existing works, the proposed method employ a simple approach for the capacity and outage analysis for receiver diversity channels operating at different signal-to-noise ratios depicted in the diagonal elements of matrix $\boldsymbol{\Omega}$. With $\mathbf{x}$ being the channel gain vector, random variable of the form $Y(a)=a+\mathbf{x}^{*} \boldsymbol{\Omega} \mathbf{x}$ is considered. Novelty of the work resides in the fact that the distribution of $Y(a)$ is accurately determined by employing Fourier representation of unit step function followed by complex integration in a straight forward way. The ergodic channel capacity is thus calculated by using the first-order moment, $E\left[\log _{2}(Y(1))\right]$, while the outage probability for a certain threshold $\gamma_{0}$ is evaluated using $\int_{0}^{\gamma_{0}} f_{Y(0)}(y) d y$. Extensive experiments have been conducted demonstrating the accuracy of the proposed approach.
\end{abstract}

Keywords: Ergodic channel capacity, Rayleigh fading, Receive diversity

\section{Introduction}

Ergodic channel capacity and outage probability are two important parameters to be considered for the design of a given communication system [1]. As such the Shannon capacity formula, initially derived for Gaussian environment, gives an upper bound for maximum transmission rate [2]. Capacity however depends on the nature of a particular channel environment. Consequently, a number of investigations have been conducted for various fading channels. Efforts have been focused to derive closed-form expressions for exact/estimated capacity [3-5].

It is well known that diversity schemes enhance the system capacity by proper utilization of random variation in a multipath wireless channel. However, the capacity evaluation and the outage analysis of diversity schemes becomes complicated. Several works have attempted to study the capacity and outage analysis of diversity channels [6,7].

\footnotetext{
*Correspondence: imrannaseem@pafkiet.edu.pk

${ }^{2}$ Department of Telecommunication Engineering, Karachi Institute of

Economics and Technology (KIET), Karachi 75190, Pakistan

Full list of author information is available at the end of the article
}

Unfortunately, the results of these works are mostly (1) approximate using some assumptions, (2) limited for some specific scenarios, and/or (3) do not result in closed form expressions. For instance, a common practice is to assume independence across the multipath channels $[6,8]$. In [9], for example, a number of closed-form expressions for channel capacity of independent multipath Rayleigh fading channels have been presented. While the assumption of independence across the channels seems appropriate enough, there are situations when such a premise is practically inadequate. Assumption of independence among diversity branches fails and there exist a correlation among them when there is insufficient antenna spacing. Consequently, the correlation between the multipath channels results in degradation of the overall performance $[10]$. In $[7,10]$, capacity with correlated fading channels have been addressed for Rayleigh and Nakagami channels, respectively. Another commonly accepted practice is to approximate a weighted sum of chi-square variables by a single one with different degrees of freedom and an adequate scaling factor. The average capacity of correlated

\section{是 Springer}

(c) 2013 Moinuddin and Naseem; licensee Springer. This is an Open Access article distributed under the terms of the Creative Commons Attribution License (http://creativecommons.org/licenses/by/2.0), which permits unrestricted use, distribution, and reproduction in any medium, provided the original work is properly cited. 
diversity Rician channels, for instance, is derived in [5] using this approximation.

To highlight the novelty of the proposed approach, some of the most important relevant works are briefly discussed below.

(a) In [7], closed-form expressions for the capacity of correlated Nakagami-m fading channels is derived. In particular, the following scenarios are considered: (i) Dual-branch maximal ratio combining, (ii) equal gain combining, (iii) selection combining, and (iv) switch and stay combining. The approach relies on the confluent hypergeometric function which results in an infinite series. The series is approximated by truncation and upper bound on the truncation error is calculated. The analysis in [7] can therefore be regarded as an approximate solution due to series truncation. Moreover, the analysis is limited to a specific scenario of equal correlation among the diversity branches.

(b) In [8], information capacity of the random signature MIMO-CDMA system is calculated. Primarily, the distribution of eigenvalues of the covariance of channel signature matrix is employed. The results, however, are limited for the scenarios of unsaturated and over saturated systems. Moreover, the methodology cannot be employed for any other MIMO system without CDMA architecture.

(c) The analysis in [9] gives closed-form expressions for the single-user capacity for Rayleigh fading channel for the MRC diversity system. Different adaptive transmission techniques are considered assuming multiple uncorrelated branches with equal average SNR. In real scenarios however, assumption of independent fading is not always true. For instance, small-size terminals with space antenna diversity may have insufficient antenna spacing to obtain independent fading in each branch. Thus, this study is limited to a specific scenario, incorporating equal average branch SNRs and equal correlation among the diversity branches.

(d) The study [10] addresses the scenario of equal average branch SNRs and arbitrary correlation between branches under three adaptive policies: (i) Optimal power and rate adaptation, (ii) constant power with optimal rate adaptation, and (iii) channel inversion with fixed rate. The approach takes into account the probability distribution function (PDF) for the sum of individual branch average SNRs (i.e., $\gamma=\sum_{i=1}^{n} \gamma_{i}$, where $n$ is the number of diversity branches). In this study, we present a novel approach of capacity and outage probability analysis of correlated diversity Rayleigh fading channels. In contrast to the above approaches, we aim to derive the PDF of a random variable of the form $Y(a)=a+\mathbf{x}^{*} \boldsymbol{\Omega} \mathbf{x}$ where $\mathbf{x}^{*}$ is Hermitian transpose of $\mathbf{x}$ [11]. Primarily, the PDF is calculated by incorporating the integration limits as a unit step function. Fourier representation of the unit step function is further used to facilitate the complex integration. Consequently, the expressions for the capacity and outage probability are evaluated using the derived PDF of $Y(a)$. Analytical results are validated through extensive experiments.

The main contributions of the proposed approach are as follows.

(a) A novel, exact, and simpler method for the capacity analysis of the correlated diversity Rayleigh channels is presented.

(b) For the purpose of unified analysis, the PDF of a generalized random variable of the form $Y(a)=a+\mathbf{x}^{*} \boldsymbol{\Omega} \mathbf{x}$ is derived using Fourier representation of the unit step function.

(c) Exact closed-form expression for the ergodic capacity of correlated diversity Rayleigh channels is evaluated for any degree of channel correlation and unequal SNRs.

(d) Exact closed form expressions for the outage probability for certain threshold $\gamma_{0}$ is evaluated.

The remainder of this article is organized as follows: Section 2 presents the problem formulation followed by the proposed approach in Section 3. Experiments are presented in Section 4 and the article is concluded in Section 5 .

\section{Ergodic capacity and outage probability of correlated diversity rayleigh fading channels}

Consider a scenario of receive diversity of index $n$, let $\mathbf{x}$ be the $n \times 1$ vector of channel gains linking the transmitter with $n$ receive antennas. Let $\boldsymbol{\Omega}$ be a diagonal matrix of order $n \times n$ such that the diagonal element $\rho_{i} ; i=$ $1,2, \ldots, n$ represents SNR of the $i$ th channel. The capacity of a diversity channel is given by [6]

$$
C=\log _{2}\left(1+\mathbf{x}^{*} \boldsymbol{\Omega} \mathbf{x}\right)
$$

Ergodic capacity is defined as $E[C]$ where $E$ is the expectation operator showing ensemble average of a random variable.

We are interested in the ergodic capacity of correlated Rayleigh fading diversity channels, the channel gain vector $\mathbf{x}$ is therefore colored circular complex Gaussian vector with zero mean and some covariance matrix say $\mathbf{R}$, that is,

$$
\mathbf{x} \sim \mathcal{C N}(\mathbf{0}, \mathbf{R})
$$


Thus, the ergodic capacity can be expressed in quadratic form $^{\mathrm{a}}$ as

$$
E[C]=E\left[\log _{2}\left(1+\mathbf{x}^{*} \boldsymbol{\Omega} \mathbf{x}\right)\right]=E\left[\log _{2}\left(1+\|\mathbf{x}\|_{\mathbf{\Omega}}^{2}\right)\right]
$$

The outage probability $P_{\text {out }}$ for a threshold $\gamma_{0}$ is defined as [12]

$$
P_{\text {out }}=\int_{0}^{\gamma_{0}} f_{\gamma}(\gamma) d \gamma
$$

where $f_{\gamma}(\gamma)$ represents the PDF of received SNR $\gamma$. The received SNR $\gamma$, for the maximum ratio combining scheme in Rayleigh fading channels, is given by

$$
\gamma=\sum_{i=1}^{n} \gamma_{i}
$$

where $\gamma_{i}$ is the instantaneous SNR of the $i$ th receive diversity branch for Rayleigh channel given by

$$
\gamma_{i}=\rho_{i}|x(i)|^{2}
$$

Thus, the combined SNR $\gamma$ at the receiver's output can be expressed as

$$
\gamma=\sum_{i=1}^{n} \rho_{i}|x(i)|^{2}=\|\mathbf{x}\|_{\boldsymbol{\Omega}}^{2}
$$

\section{The proposed approach}

First defining the following random variable:

$$
Y(a) \triangleq a+\mathbf{x}^{*} \mathbf{\Omega} \mathbf{x}
$$

It can be seen that the ergodic capacity given in (3) can be obtained by choosing $a=1$, that is,

$$
E[C]=E\left[\log _{2} Y(1)\right]=\int_{0}^{\infty} \log _{2}(y) f_{Y(1)}(y) d y
$$

Similarly, the combined SNR given in (7) can be computed with the choice of $a=0$, that is,

$$
\gamma=Y(0)
$$

and consequently, the outage probability can be determined as

$$
P_{\text {out }}=\int_{0}^{\gamma_{0}} f_{Y(0)}(y) d y
$$

Thus, our approach relies on evaluating the PDF and the moment of the random variable of the quadratic form $Y(a)=a+\|\mathbf{x}\|_{\mathbf{D}}^{2}$ where $\mathbf{D}$ is a diagonal matrix. Next, we present the derivation for the PDF of random variable $Y(a)$.

\subsection{The PDF of $Y(a)$}

The random variable $Y$ defined in (8) can be rewritten in quadratic form as

$$
Y(a)=a+\|\mathbf{x}\|_{\Omega}^{2}
$$

It can easily be shown that the quadratic term $\|\mathbf{x}\|_{\mathbf{\Omega}}^{2}$ can equivalently be written as $\left\|\mathbf{x}_{w}\right\|_{\mathbf{\Omega} \mathbf{R}}^{2}$ where $\mathbf{x}_{w}$ is the whitened version of $\mathbf{x}$, i.e., $\mathbf{x}_{w} \sim \mathcal{C N}(\mathbf{0}, \mathbf{I})$, where $\mathbf{I}$ is the identity matrix. As a result, the random variable $Y(a)$ can be setup as follows

$$
Y(a)=a+\left\|\mathbf{x}_{w}\right\|_{\mathbf{\Omega} \mathbf{R}}^{2}
$$

By definition, the $\mathrm{CDF}$ of $Y(a)$ is given as

$$
F_{Y(a)}(y)=P[Y(a) \leq y]
$$

which can be rewritten as

$$
\begin{aligned}
F_{Y(a)}(y) & =P\left[a+\left\|\mathbf{x}_{w}\right\|_{\mathbf{\Omega} \mathbf{R}}^{2} \leq y\right] \\
& =P\left[y-a-\left\|\mathbf{x}_{w}\right\|_{\mathbf{\Omega} \mathbf{R}}^{2} \geq 0\right]
\end{aligned}
$$

Now, by using the definition of unit step function, the CDF of $Y(a)$ becomes

$$
F_{Y(a)}(y)=\int_{-\infty}^{\infty} p\left(\mathbf{x}_{w}\right) u\left(y-a-\left\|\mathbf{x}_{w}\right\|_{\mathbf{\Omega} \mathbf{R}}^{2}\right) d \mathbf{x}_{w}
$$

using Fourier representation of the unit step function

$$
u(x)=\frac{1}{2 \pi} \int_{-\infty}^{\infty} \frac{e^{x(j \omega+\beta)}}{j \omega+\beta} d \omega ; \beta>0
$$

the $\mathrm{CDF}$ of $Y$ becomes

$$
\begin{aligned}
F_{Y(a)}(y)= & \frac{1}{2 \pi^{n+1}} \int_{-\infty}^{\infty} e^{-\mathbf{x}_{w}^{*} \mathbf{x}_{w}} \\
& \times \int_{-\infty}^{\infty} \frac{e^{\left(y-\left\|\mathbf{x}_{w}\right\|_{\Omega \mathbf{R}}^{2}-a\right)(j \omega+\beta)}}{j \omega+\beta} d \omega d \mathbf{x}_{w}
\end{aligned}
$$

After rearranging the similar terms, we obtain

$$
\begin{aligned}
F_{Y(a)}(y)= & \frac{1}{2 \pi^{n+1}} \int_{-\infty}^{\infty} \int_{-\infty}^{\infty} e^{-\mathbf{x}_{w}^{*}[\mathbf{I}+(j \omega+\beta) \boldsymbol{\Omega} \mathbf{R}] \mathbf{x}_{w}} d \mathbf{x}_{w} \\
& \times \frac{e^{(y-a)(j \omega+\beta)}}{j \omega+\beta} d \omega
\end{aligned}
$$

The inner integral in (19) above can be solved using eigenvalue decomposition $\mathbf{R}=\mathbf{Q} \Lambda \mathbf{Q}^{*}$ where $\mathbf{Q}$ is a matrix of eigenvectors of matrix $\mathbf{R}$ and $\Lambda$ is a diagonal matrix with $\lambda_{i}=\Lambda(i, i)$ corresponding to the $i$ th eigenvalue of $\mathbf{R}$. Thus, by applying this eigenvalue decomposition and using a change of variable $\tilde{\mathbf{x}}_{w}=\mathbf{Q}^{*} \mathbf{x}_{w}$, the inner integral in (19) can be set up as

$$
\frac{1}{\pi^{n}} \int_{-\infty}^{\infty} e^{-\tilde{\mathbf{x}}_{w}^{*}[\mathbf{I}+\boldsymbol{\Lambda}(\beta+j \omega) \boldsymbol{\Omega}] \tilde{\mathbf{x}}_{w}} d \tilde{\mathbf{x}}_{w}
$$

The above expression is merely a Gaussian integral and can therefore be reduced to

$$
\frac{1}{\prod_{i=1}^{n}\left(1+\lambda_{i}(j \omega+\beta) \rho_{i}\right)}
$$

As a result, the CDF given in (19) takes the form

$$
F_{Y(a)}(y)=\int_{-\infty}^{\infty} \frac{e^{j \omega y-j \omega a+y \beta-a \beta}}{2 \pi(j \omega+\beta) \prod_{i=1}^{n}\left(1+\lambda_{i}(j \omega+\beta) \rho_{i}\right)} d \omega
$$


The PDF of $y$ is obtained by differentiating Equation (20)

$$
f_{Y(a)}(y)=\frac{e^{\beta(y-a)}}{2 \pi} \int_{-\infty}^{\infty} \frac{e^{j \omega(y-a)}}{\prod_{i=1}^{n}\left(1+\lambda_{i}(j \omega+\beta) \rho_{i}\right)} d \omega
$$

Considering the scenario of unique eigenvalues of $\mathbf{R}$, fraction in Equation (21) can be decomposed as

$$
\begin{aligned}
\frac{1}{\prod_{i=1}^{n} 1+\lambda_{i}(j \omega+\beta) \rho_{i}}= & \frac{\alpha_{1}}{1+\lambda_{1}(j \omega+\beta) \rho_{1}} \\
& +\frac{\alpha_{2}}{1+\lambda_{2}(j \omega+\beta) \rho_{2}}+\cdots
\end{aligned}
$$

it is straightforward to note that

$$
\alpha_{l}=\frac{1}{\prod_{i=1, i \neq l}^{n}\left(1-\frac{\rho_{i} \lambda_{i}}{\rho_{l} \lambda_{l}}\right)}
$$

Thus, the PDF in (21) can be written as

$$
f_{Y(a)}(y)=\frac{e^{\beta(y-a)}}{2 \pi} \sum_{l=1}^{n} \int_{-\infty}^{\infty} \frac{\alpha_{l} e^{j \omega(y-a)}}{1+\lambda_{l}(j \omega+\beta) \rho_{l}} d \omega
$$

The integral appearing in (24) can be solved using the following solution from residue theory [13]

$$
\frac{1}{2 \pi} \int_{-\infty}^{\infty}(\mu+j x)^{-v} e^{-j p x} d x=\frac{(-p)^{\nu-1} e^{\mu p}}{\Gamma(\nu)} u(-p)
$$

which is valid for $\operatorname{Re}(\nu)>0$ and $\operatorname{Re}(\mu)>0$. Thus, $\mathrm{PDF}$ of $Y(a)$ is found to be

$$
f_{Y(a)}(y)=\sum_{l=1}^{n} \frac{e^{-\left(\frac{y-a}{\lambda_{l} \rho_{l}}\right)}}{\rho_{l} \lambda_{l} \prod_{i=1, i \neq l}^{n}\left(1-\frac{\rho_{i} \lambda_{i}}{\rho_{l} \lambda_{l}}\right)} u(y-a)
$$

\subsection{Evaluation of ergodic capacity}

For the calculation of ergodic capacity, substituting the PDF of $Y(a)$ with $a=1$ from (26) into (9). Thus,

$$
\begin{aligned}
E[C] & =\int_{0}^{\infty} \log _{2}(y) f_{Y(1)}(y) d y \\
& =\int_{-\infty}^{\infty} \log _{2}(y) \sum_{l=1}^{n} \frac{e^{-\left(\frac{y-1}{\lambda_{l} \rho_{l}}\right)}}{\rho \lambda_{l} \prod_{i=1, i \neq l}^{n}\left(1-\frac{\lambda_{i}}{\lambda_{l}}\right)} u(y-1) d y \\
& =\log _{2} e \sum_{l=1}^{n} \frac{e^{\frac{1}{\lambda_{l} \rho_{l}}}}{\rho_{l} \lambda_{l} \prod_{i=1, i \neq l}^{n}\left(1-\frac{\rho_{i} \lambda_{i}}{\rho_{l} \lambda_{l}}\right)} \int_{1}^{\infty} \ln (y) e^{\frac{-y}{\lambda^{l} \rho_{l}}} d y
\end{aligned}
$$

Finally, by solving the above integral, the ergodic capacity is found to be

$$
E[C]=-\log _{2} e \sum_{l=1}^{n}\left[\frac{e^{\frac{1}{\lambda_{l} \rho_{l}}}}{\prod_{i=1, i \neq l}^{n}\left(1-\frac{\rho_{i} \lambda_{i}}{\rho_{l} \lambda_{l}}\right)} E_{i}\left(\frac{-1}{\lambda_{l} \rho_{l}}\right)\right]
$$

where $E_{i}($.$) is the Exponential Integral function defined as$ [13]:

$$
E_{i}(x) \triangleq \begin{cases}\int_{-\infty}^{x} \frac{e^{t}}{t} d t, & x<0 \\ e^{x}\left[\frac{1}{x}+\int_{0}^{\infty} \frac{e^{-t}}{(x-t)^{2}} d t\right], & x>0\end{cases}
$$

\subsection{Evaluation of outage probability}

Similarly, the outage probability can be computed by substituting the PDF of $Y(a)$ with $a=0$ from (26) into (11). Thus, it is found that

$$
\begin{aligned}
P_{\text {out }} & =\int_{0}^{\gamma_{0}} f_{Y(0)}(y) d y \\
& =\int_{0}^{\gamma_{0}} \sum_{l=1}^{n} \frac{e^{-\left(\frac{y}{\lambda_{l} \rho_{l}}\right)}}{\rho_{l} \lambda_{l} \prod_{i=1, i \neq l}^{n}\left(1-\frac{\rho_{i} \lambda_{i}}{\rho_{l} \lambda_{l}}\right)} u(y) d y \\
& =\sum_{l=1}^{n} \frac{1}{\rho_{l} \lambda_{l} \prod_{i=1, i \neq l}^{n}\left(1-\frac{\rho_{i} \lambda_{i}}{\rho_{l} \lambda_{l}}\right)} \int_{0}^{\gamma_{0}} e^{-\left(\frac{y}{\lambda_{l} \rho_{l}}\right)} d y \\
& =\sum_{l=1}^{n} \frac{\left(1-e^{-\left(\frac{\gamma_{0}}{\lambda_{l} \rho_{l}}\right)}\right)}{\prod_{i=1, i \neq l}^{n}\left(1-\frac{\rho_{i} \lambda_{i}}{\rho_{l} \lambda_{l}}\right)}
\end{aligned}
$$

\section{Experimental results}

Extensive experiments were carried out to investigate the validity of the proposed approach. With the receive antennas having diversity order $n$, the channel correlation matrix $\mathbf{R}$ with correlation coefficient $\gamma_{c}$ is given by

$$
\mathbf{R}=\left[\begin{array}{ccccc}
1 & \gamma_{c} & \gamma_{c}^{2} & \cdots & \gamma_{c}^{n-1} \\
\gamma_{c} & 1 & \gamma_{c} & \cdots & \gamma_{c}^{n-2} \\
\gamma_{c}^{2} & \gamma_{c} & 1 & \cdots & \gamma_{c}^{n-3} \\
\vdots & & & & \\
\gamma_{c}^{n-1} & \gamma_{c}^{n-2} & \gamma_{c}^{n-3} & \cdots & 1
\end{array}\right]
$$

The experiments were aimed to investigate: (1) effect of correlation coefficient $\left(\gamma_{c}\right)$ on the channel capacity; (2) variation in channel capacity with respect to the change in diversity order $n$; (3) effect of the SNR on the channel capacity; (4) effect of the SNR on the Outage Probability; (5) effect of correlation coefficient on the outage probability; and (6) agreement of simulation and analytical results for all sets of experiments.

The first set of experiments is targeted to evaluate the performance with respect to the diversity order $n$. The results are shown in Figure 1. Two extreme cases of $\gamma_{c}=$ 0.2 and $\gamma_{c}=0.8$ are considered. For all the experiments we design the matrix $\boldsymbol{\Omega}$ such that $\rho_{i}=i \mathrm{~dB} ; i=1,2, \ldots, n$. For a comprehensive comparison, performance curves are obtained for Equation (1) using Monte Carlo method, each simulated value $E[C]$ is the result of averaging over 4,000 independent computer trials. 


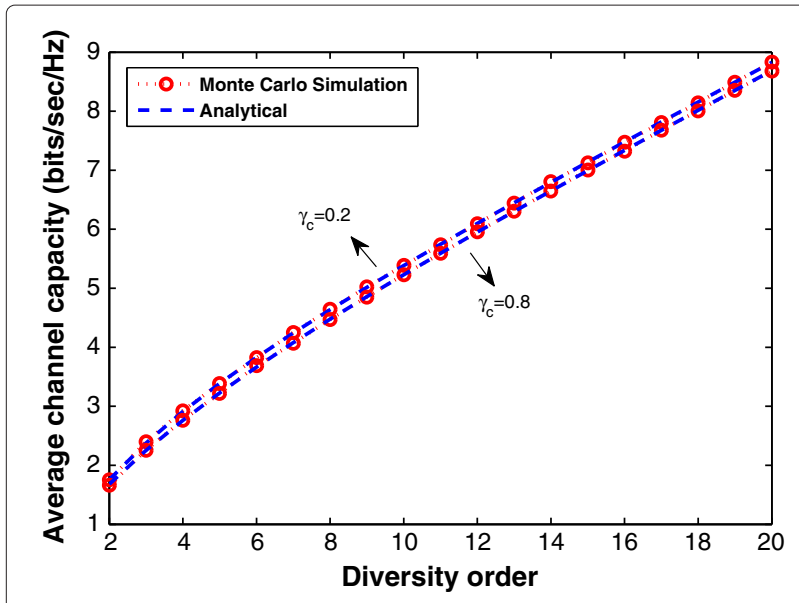

Figure 1 Capacity of a Rayleigh channel with respect to increasing diversity order.

These are referred to as simulation results, analytical results are attained using the exact capacity expression derived in Equation (28). Performance curves are shown in Figure 1. With an increase in the correlation coefficient, slight decrease in the channel capacity is observed, whereas the performance increases substantially with an increase in the diversity order. As such the simulation and analytical values are quite identical. For both cases of $\gamma_{c}=$

Table 1 Comparison of simulation and analytical results for the case study $\gamma_{c}=0.2$

\begin{tabular}{cccc}
\hline Diversity order $(\boldsymbol{n})$ & Simulation & Analytical & Relative error \\
\hline 2 & 1.7523 & 1.7579 & 0.0056 \\
3 & 2.4006 & 2.3768 & 0.0238 \\
4 & 2.9253 & 2.9104 & 0.0150 \\
5 & 3.3848 & 3.3883 & 0.0035 \\
6 & 3.8236 & 3.8283 & 0.0046 \\
7 & 4.2489 & 4.2415 & 0.0074 \\
8 & 4.6411 & 4.6354 & 0.0057 \\
9 & 5.0210 & 5.0151 & 0.0059 \\
10 & 5.3878 & 5.3840 & 0.0038 \\
11 & 5.7345 & 5.7448 & 0.0103 \\
12 & 6.0926 & 6.0994 & 0.0067 \\
13 & 6.4468 & 6.4491 & 0.0023 \\
14 & 6.8037 & 6.7951 & 0.0086 \\
15 & 7.1281 & 7.1382 & 0.0101 \\
16 & 7.4709 & 7.4790 & 0.0080 \\
17 & 7.8066 & 7.8179 & 0.0113 \\
18 & 8.1453 & 8.1555 & 0.0102 \\
19 & 8.4927 & 8.4920 & 0.0007 \\
20 & 8.8326 & 8.8275 & 0.0051 \\
\hline
\end{tabular}

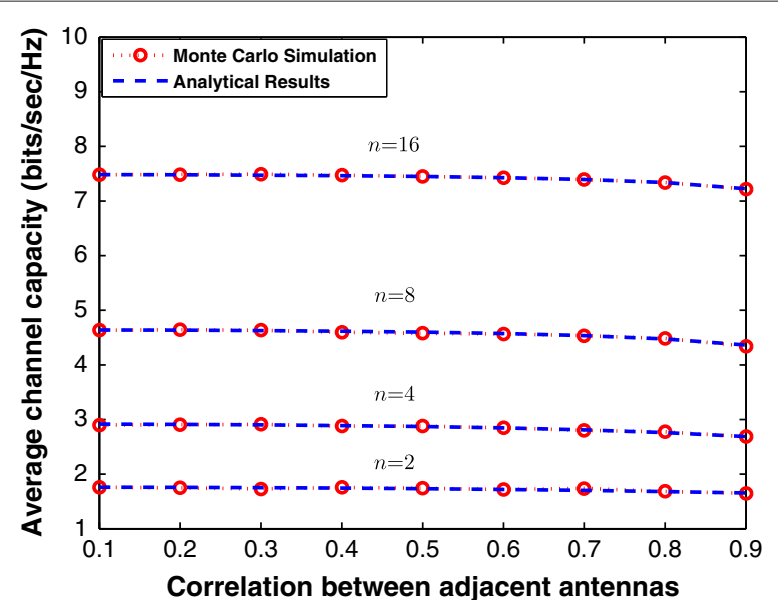

Figure 2 Capacity of a Rayleigh channel with respect to the correlation between adjacent antennas. Note the gain in capacity with the increasing diversity order.

0.2 and $\gamma_{c}=0.8$, the curves for analytical and simulation values almost overlap each other. A detailed comparison for the case study of $\gamma_{c}=0.2$ is dilated in Table 1. For all values of $n$, the relative error between the simulated and analytical values is quite low, as such the minimum error of 0.0007 is reported for the system with 19 receive antennas.

The second set of experiments was aimed to highlight the significance of the correlation coefficient, $\gamma_{c}$, for different diversity orders. Results are shown in Figure 2, various diversity orders of $2,4,8$, and 16 were considered. The performance curves obtained across the whole range $\gamma_{c}=0.1-0.9$ clearly show a degradation with the increase of the correlation coefficient. The analytical values are quite similar to their simulated counterparts. Table 2 shows results for the case with $n=16$. As such the minimum relative error of 0.0012 is reported for the case $\gamma_{c}=0.6$.

Table 2 Capacity comparison with respect to variation in the correlation coefficient with $n=16$

\begin{tabular}{lccc}
\hline $\boldsymbol{\gamma}_{\boldsymbol{c}}$ & Simulation & Analytical & Relative error \\
\hline 0.1 & 2.8994 & 2.9153 & 0.0159 \\
0.2 & 2.9061 & 2.9104 & 0.0043 \\
0.3 & 2.9095 & 2.9019 & 0.0077 \\
0.4 & 2.8791 & 2.8891 & 0.0099 \\
0.5 & 2.8798 & 2.8709 & 0.0089 \\
0.6 & 2.847 & 2.8458 & 0.0012 \\
0.7 & 2.8006 & 2.8107 & 0.0101 \\
0.8 & 2.7752 & 2.7610 & 0.0142 \\
0.9 & 2.689 & 2.6867 & 0.0023 \\
\hline
\end{tabular}




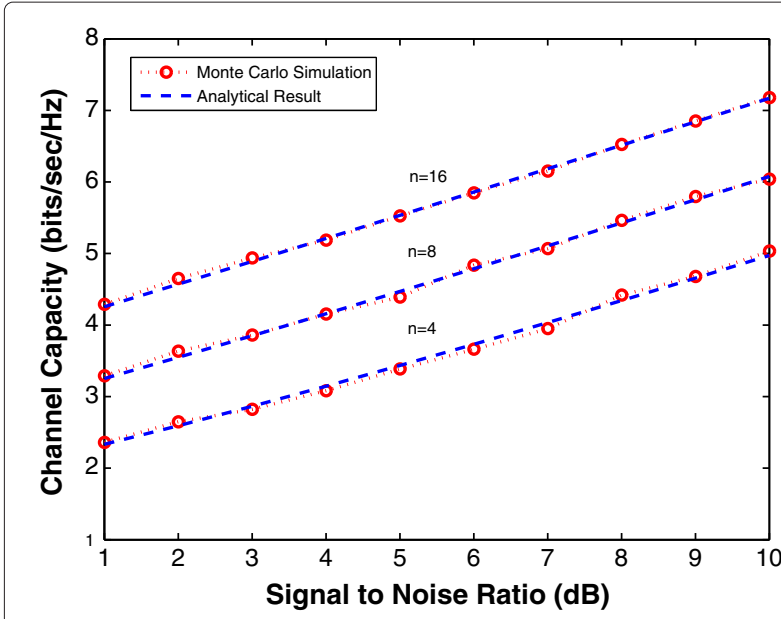

Figure 3 Influence of the transmitter SNR on channel capacity. Experiments were conducted with high correlation coefficient $\gamma_{c}=0.8$

Third set of experiments was targeted to investigate the effect of the SNR. For this particular evaluation, we assume that all channels have equal SNR values, experiments were conducted at $\gamma_{c}=0.8$ and results are illustrated in Figure 3. Experiments were conducted with various diversity orders of 4,8 , and 16 , as expected the channel capacity increases with the increase in the SNR. Again, the analytical results are found to be quite similar to the simulation values, results for the case $n=8$ are dilated in Table 3. As such no error is reported for the case of 9-dB SNR where analytical result is in exact agreement with the simulated value.

Next, the effect of SNR on the outage probability with equal branch SNRs $\left(\rho_{i}\right)$ is investigated. The outage probability is calculated with $\gamma_{c}=0.5$ for different values of SNRs on a wide rang of threshold $\gamma_{0}$. Four different values of SNR, namely $5,10,15$, and $20 \mathrm{~dB}$, are used in the experiment and the results are reported in Figure 4. It can

Table 3 Capacity comparison for simulation and analytical experiments with $n=8$

\begin{tabular}{cccc}
\hline SNR (dB) & Simulation & Analytical & Relative error \\
\hline 1 & 3.2437 & 3.2523 & 0.0086 \\
2 & 3.5371 & 3.5479 & 0.0108 \\
3 & 3.9269 & 3.8502 & 0.0768 \\
4 & 4.1549 & 4.1579 & 0.0030 \\
5 & 4.4301 & 4.4703 & 0.0402 \\
6 & 4.7859 & 4.7865 & 0.0006 \\
7 & 5.1043 & 5.1058 & 0.0015 \\
8 & 5.3680 & 5.4276 & 0.0597 \\
9 & 5.7515 & 5.7515 & 0.0000 \\
10 & 6.1104 & 6.0770 & 0.0334 \\
\hline
\end{tabular}

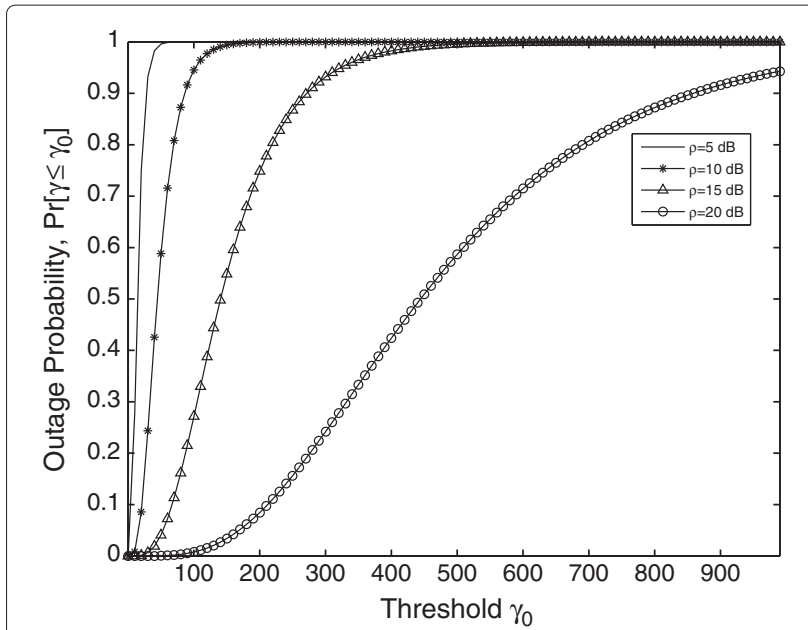

Figure 4 Outage probability versus threshold $\gamma_{0}$ for different values of average branch SNR $\rho$.

be depicted from the figure that the outage probability at a certain threshold value decreases by increasing the SNR.

Finally, the effect of channel correlation coefficient $\gamma_{c}$ on the outage probability with equal branch SNRs of $20 \mathrm{~dB}$ is investigated. For a comprehensive analysis, three different values of the correlation coefficient, i.e., 0.01, 0.5, and 0.99, are chosen constituting three different experiments. The outage probability is calculated for a wide rang of threshold $\gamma_{0}$. The results depicted in Figure 5 show that the outage probability is maximum for $\gamma_{c}=0.01$ before the value of $\gamma_{0}$ approaches 580 (approximately). Beyond this point the outage probability is maximum for $\gamma_{c}=0.99$.

\section{Conclusion}

A novel and exact expression for average capacity of correlated diversity Rayleigh fading channels is presented. The proposed approach relies on exact evaluation of the

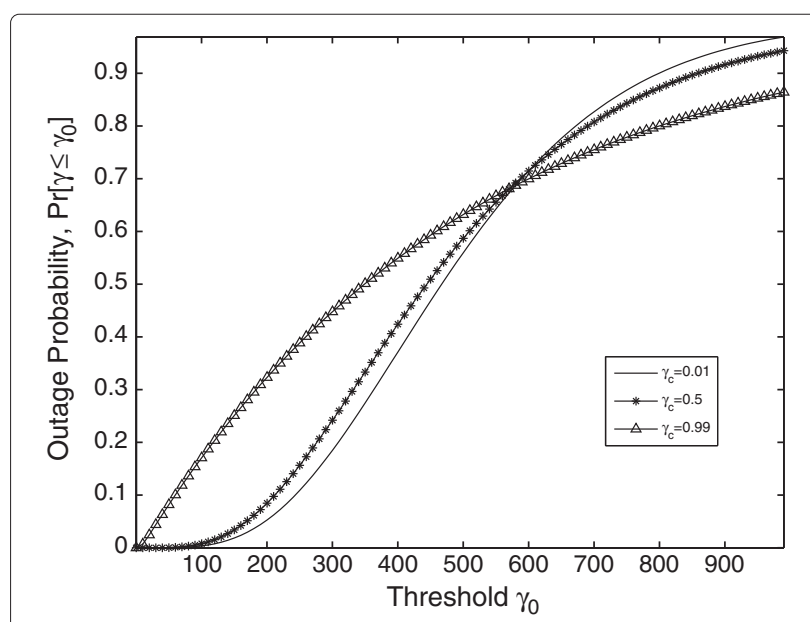

Figure 5 Outage probability versus threshold $\gamma_{0}$ for different values of correlation coefficient $\gamma_{c}$. 
CDF of random variable of the form $1+\mathbf{x}^{*} \boldsymbol{\Omega} \mathbf{x}$. This is essentially achieved by using Fourier representation of the unit step function followed by complex integration. The main contribution of the proposed research is the exact analysis in a simpler way which avoids approximations and sophisticated expressions. Extensive experiments have been conducted to investigate the accuracy of the proposed approach. Analytical and simulation results are found to be in agreement for all sets of experiments.

\section{Endnote}

${ }^{a}$ For any matrix $\mathbf{D}$, the quadratic form $\|\mathbf{v}\|_{\mathbf{D}}^{2}$ is defined as $\|\mathbf{v}\|_{\mathbf{D}}^{2} \triangleq \mathbf{v}^{*} \mathbf{D} \mathbf{v}$.

\section{Competing interests}

The authors declare that they have no competing interests.

\section{Author details}

${ }^{1}$ Telecommunication Engineering Department, Iqra University, Karachi, Pakistan. ${ }^{2}$ Department of Telecommunication Engineering, Karachi Institute of Economics and Technology (KIET), Karachi 75190, Pakistan.

Received: 6 May 2012 Accepted: 10 January 2013

Published: 30 January 2013

\section{References}

1. NC Sagias, GS Tombras, GK Karagiannidis, New results for the Shannon channel capacity in generalized fading channels. IEEE Commun. Lett. 9(2), 97-99 (2005)

2. DB Costa, MD Yacoub, Average channel capacity for generalized fading scenarios. IEEE Commun. Lett. 11(12), 949-951 (2007)

3. WCY Lee, Estimate of channel capacity in Rayleigh fading environment. IEEE Trans. Veh. Technol. 39(3), 187-189 (1990)

4. CG Günther, Comments on "Estimate of channel capacity in Rayleigh fading environment". IEEE Trans. Veh. Technol. 45(2), 401-403 (1996)

5. QT Zhang, DP Liu, A simple capacity formula for correlated diversity Rician fading channels. IEEE Commun. Lett. 6(11), 481-483 (2002)

6. E Telatar, Capacity of multi-antenna Gaussian channels. AT\&T Labs, BLO 112 170-950 615-07TM, 1995

7. S Khatalin, JP Fonseka, Capacity of correlated Nakagami-m fading channels with diversity combining techniques. IEEE Trans. Veh. Technol. 55, 142-150 (2006)

8. PB Rapajic, D Popescu, Information capacity of a random signature multiple-input multiple-output channel. IEEE Trans. Commun. 48(8), 1245-1248 (2000)

9. M Alouini, A Goldsmith, Capacity of Rayleigh fading channels under different adaptive transmission and diversity combined techniques. IEEE Trans. Veh. Technol. 48(4), 1165-1181 (1999)

10. RK Malik, MZ Win, JW Shao, M Alouini, A Goldsmith, Channel capacity of adaptive transmission with maximal ratio combining in correlated Rayleigh fading channel. IEEE Trans. Wirel. Commun. 3(4), 1124-1133 (2004)

11. TY Al-Naffouri, M Moinuddin, Exact performance analysis of the $\epsilon$-NLMS algorithm for colored circular Gaussian inputs. IEEE Trans. Signal Process. 58(10), 5080-5090 (2010)

12. J Proakis, Digital Communications (McGraw-Hill, New York, 2000)

13. IS Gradshteyn, IM Ryzhik, Table of Integral, Series and Products, Corrected and Enlarged Edition (Academic Press, Inc., New York, 1980)

doi:10.1186/1687-1499-2013-20

Cite this article as: Moinuddin and Naseem: A simple approach to evaluate the ergodic capacity and outage probability of correlated Rayleigh diversity channels with unequal signal-to-noise ratios. EURASIP Journal on Wireless Communications and Networking 2013 2013:20.

\section{Submit your manuscript to a SpringerOpen ${ }^{\circ}$ journal and benefit from:}

- Convenient online submission

- Rigorous peer review

- Immediate publication on acceptance

- Open access: articles freely available online

- High visibility within the field

- Retaining the copyright to your article

Submit your next manuscript at $>$ springeropen.com 